\title{
Host eyes, brains and their brood parasites
}

\author{
Anders Møller ${ }^{1}$ and Johannes Erritzøe ${ }^{2}$ \\ ${ }^{1}$ Univ. Paris-Sud \\ ${ }^{2}$ Taps Old Rectory
}

October 28,2021

\begin{abstract}
1. Brood parasites interact with their hosts for exploitation of host parental abilities and the associated resources. This results in coevolutionary interactions of hosts and parasites. 2. A prime example of such a common specialist brood parasite is the common cuckoo Cuculus canorus and its host races. Hosts use their cognitive abilities to identify parasites and vice versa for their ability to discriminate among potential hosts. 3. We predicted that parasites with relatively large brains for their body size should be more successful at avoiding their hosts, and that hosts with small brains for their body size should more often be exploited by parasites. We also predicted that hosts with relatively large eyes for their body size would have superior discriminatory abilities allowing for superior discrimination against brood parasites. Finally, we predicted that visual ability of specific cuckoo hosts would have evolved exaggerated visual ability as estimated from the relative size of their optic tectum would have resulted in such hosts being more successful as reflected by their higher rate of parasitism. 4. Interspecific variation in size of brain, eye, optic tectum, telencephalon and cerebellum were consistent with these predictions.
\end{abstract}

\section{Hosted file}

Host eyes, brains_18092021.docx available at https://authorea.com/users/443402/articles/ 543389-host-eyes-brains-and-their-brood-parasites 